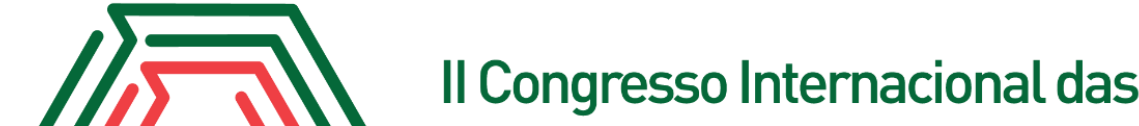 Ciências Agrárias COINTER - PDVAgro 2017
}

\section{DESENVOLVIMENTO INICIAL DE MUDAS DE PAU PRETO EM DIFERENTES SUBSTRATOS.}

Apresentação: Comunicação Oral

\section{Phiama Katryna M. De Azevedo ${ }^{1}$; Beatriz Chaves da Silva ${ }^{2}$; Suelen Melo De Oliveira ${ }^{3}$; Ivonete Araújo Gonçalves ${ }^{4}$; Mônica Trindade Abreu de Gusmão ${ }^{5}$. \\ RESUMO}

Cenostigma tocantinum Ducke é uma espécie da família Fabaceae e subfamília Caesalpiniaceae, sendo encontrada no Estado do Pará, na região do Rio Tocantins, com a denominação de Pau-preto. Geralmente utilizada como arborização, por possuir características de paisagismo e beleza. Objetivou-se neste trabalho avaliar o desenvolvimento inicial de mudas de Pau preto (C. tocantinum Ducke), em diferentes substratos. O experimento teve duração de Fevereiro a Maio de 2016, e a procedência das sementes utilizadas no estudo é da localidade UFRA, Campus Belém/PA. Sendo utilizadas no trabalho sementes retiradas manualmente do fruto no período de fevereiro de 2016. Após a coleta dos frutos foi realizada uma abertura manual e o beneficiamento das sementes e foi conduzido em viveiro, em delineamento em blocos ao acaso, com cinco tratamentos e com cinco repetições, sendo que cada parcela foi composta por 12 plantas e a área útil composta por 5 plantas, totalizando 300 plantas, aplicados aos tratamentos TP, TP+SC 2:1, TP+SC 3:1, TP+FC 2:1, TP+FC 3:1, Onde TP: Terra Preta, TP+SC: Terra Preta+Serragem Curtida e TP+FC: Terra Preta + Fibra de Coco. Analisou-se a taxa de germinação (TG\%) e índice de velocidade de germinação (IVG) das sementes coletadas, além do crescimento em altura $(\mathrm{m})$ e diâmetro a altura do coleto DAC (mm) das mudas, a contagem do número de folhas emitidas. Os resultados mostraram que T1, serragem curtida $2: 1$ foi o que mais se destacou, com melhor qualidade e maior porcentagem de germinação das mudas, os indivíduos na qual se obteve baixos índices de germinação foi o T0, a testemunha.

PALAVRAS-CHAVE: Cenostigma tocantinum Ducke; Germinação; Crescimento de mudas.

\footnotetext{
${ }^{1}$ Graduanda de engenharia Florestal, Universidade Federal Rural da Amazônia, phiamaufra@hotmail.com

${ }^{2}$ Graduanda de engenharia Florestal, Universidade Federal Rural da Amazônia, biachavees@ gmail.com

${ }^{3}$ Graduanda de engenharia Florestal, Universidade Federal Rural da Amazônia, ssuelenmelo@gmail.com

${ }^{4}$ Graduanda de engenharia Florestal, Universidade Federal Rural da Amazônia, Ivonete.araujo.g@gmail.com

5. Professora da Universidade Federal Rural da Amazônia (UFRA), monica.gusmao@ufra.edu.br
} 


\section{Introdução}

Gusmão M. T. A.; Ferreira G. C.; Ohashi S. T. relata que Cenostigma tocantinum Ducke é a família Fabacea e subfamília Caesalpinoideae tem nomes vulgares como pau-preto, pau-pretinho, cassia rodoviária, mangiribá. Tem ocorrência nos estados do Pará, Maranhão, Amapá, Amazonas, Rondônia, Goiás, Bahia, e Tocantins.

Além do seu grande potencial para arborização, esta espécie florestal também apresenta importância econômica na construção civil, como caibros, ripas e vigas, além de sua utilidade em obras externas por apresentar madeira bastante durável quando exposta.

De Sousa S. G. A.; Moraes R. P.; Garcia L. C. diz que pau-preto atualmente vem sendo usado com maior frequência na arborização de ruas, avenidas, praças e parques. Entretanto, apesar de todas as potencialidades e vantagens atribuídas a essa espécie, faltam estudos sobre vários aspectos.

Segundo Garcia (2008), por ser uma espécie nativa da Amazônia, apresenta baixa suscetibilidade ao ataque de pragas e doenças e sobre as sementes a longevidade natural é um aspecto importante.

A germinação corresponde a uma etapa na vida da semente que é influenciada por fatores ambientais e genéticos durante o seu desenvolvimento. A germinação das sementes de Pau preto tem sido objeto de estudos visando atender a grande demanda de mudas para diversos tipos de projetos. ( Lopes e Pereira ;2005)

Estudar o comportamento das sementes em relação aos diferentes tipos de substrato se torna importante, pois fomenta a pesquisa, auxilia empresas, acadêmicos e aqueles que utilizarão a espécie estudada. (GARCIA, 2008).

$\mathrm{O}$ alto grau de umidade das sementes é uma das principais causas da perda do poder germinativo durante o armazenamento. Este fato ocasiona o aumento da taxa respiratória e a ação de microorganismos, sendo que graus de umidade superiores a $20 \%$ podem promover o aquecimento da massa de sementes a uma temperatura letal (Desai et al., 1997).

O tipo de substrato é importante na formação e germinação da muda (Peixoto, 1986). Os melhores substratos devem apresentar, entre outras importantes características, fácil disponibilidade de aquisição e transporte, ausência de patógenos, riqueza em nutrientes essenciais, pH adequado, boa textura e estrutura (Silva et al., 2001).

O substrato influência no comportamento germinativo das espécies de maneira não uniforme, de forma que algumas são mais exigentes e com melhor desempenho em apenas um tipo de substrato ou pela mistura deste. Para Souza et al. (2001) é o responsável pelo fornecimento de nutrientes, disponibilidade e capacidade de retenção de água. 
A prática de cultivar plantas utilizando substratos objetiva a determinação do melhor padrão vital de cultivo no menor tempo, nesse sentido, a sua escolha ocorre em função da facilidade e da eficiência do uso e do tipo de semente, pois, é onde o sistema radicular contribuirá no crescimento da parte aérea até o transplantio (Braga Junior et al. 2010; Jabur; Martins, 2002).

Neste sentido, o presente trabalho tem como objetivo avaliar o desenvolvimento inicial de mudas de pau preto (Cenostigma tocantinum Ducke), em diferentes substratos.

\section{Fundamentação Teórica}

A produção de mudas de espécies nativas florestais ainda é muito escassa, com isso há uma necessidade de conhecimento nesta area, conhecimento em tecnologias, novos substratos, ao qual proporciona um crescimento mais rápido, pois com a técnica certa e substrato adequado se tem mudas mais vigorosas, os testes são importantes, pois proporciona o conhecimento prévio em qual substrato há um melhor desenvolvimento e onde implanta-las.

\section{Material e métodos}

\section{Área de estudo}

O experimento foi conduzido em um viveiro com sombrite a 50\%, mais especificamente no Instituto de Ciências Agrárias (ICA), da Universidade Federal Rural da Amazônia (UFRA), campus Belém/PA.

O município apresenta clima quente e úmido com precipitação média anual alcançando os $2.834 \mathrm{~mm}$ e temperatura média de $26^{\circ} \mathrm{C}$ (INMET, 2012). Segundo SANTOS et al. (1983) a Universidade Federal Rural da Amazônia - UFRA, local da coleta das sementes e do experimento, não possui períodos secos definidos, tem como topografia o relevo plano, e em sua cobertura vegetal há predominância de latifólias secundárias e de gramíneas. Os solos mapeados na área pelos autores variaram entre: Latossolo Amarelo Álico; Concrecionário Laterítico Álico, Plintossolos álico; Glei pouco húmicos eutróficos e distróficos; e os Hidromórficos Indiscriminados, esses dois últimos compõe os solos das várzeas do Rio Guamá.

\section{Coleta das sementes}

O experimento teve duração de Fevereiro a Maio de 2016, e a procedência das sementes utilizadas no estudo é da localidade UFRA, Campus Belém/PA. Sendo utilizadas no trabalho sementes retiradas manualmente do fruto no período de fevereiro de 2016. Após a coleta dos frutos foi realizada uma abertura manual e $\mathrm{O}$ beneficiamento das sementes. Inicialmente as 
sementes de C.tocantinum Ducke foram submetidas à embebição em água destilada em um período de 24 horas.

A semeadura foi feita pelo método de semeio direto em recipientes de sacos de polietileno com $15 \mathrm{~cm}$ de diâmetro e $20 \mathrm{~cm}$ de altura, onde foi semeada uma semente por recipiente, em uma profundidade adequada para semente, mantidos com níveis de sombreamento (sombrites 50\%), contendo uma mistura de substrato conforme especificado no item Tratamento e delineamento experimental, e identificados com plaquetas contendo data e tipo de tratamento

\section{Implantação do experimento}

Realizamos a limpeza e a restauração do canteiro telado com dimensão $10 \mathrm{mx} 1,20 \mathrm{~m}$ totalizando $12 \mathrm{~m}^{2}$, onde observamos a existência de uma grande quantidade de sauveiros ao redor. Combatemos com Mirex em isca, onde foi colocado no solo em seis pontos estratégicos, sendo o formicida coberto por uma garrafa pet cortado ao meio.

E em seguida alocamos os sacos de mudas no canteiro com intensidade luminosa controlada e fixada em 50\%, montando o experimento obedecendo aos critérios do delineamento de blocos ao acaso. Os 300 sacos de mudas foram distribuídos, em uma área de $6,30 \mathrm{~m} \times 1,20 \mathrm{~m}$, com espaçamento entre blocos de 0,50cm. (Figura 3 )

Figura 3: modelo do canteiro

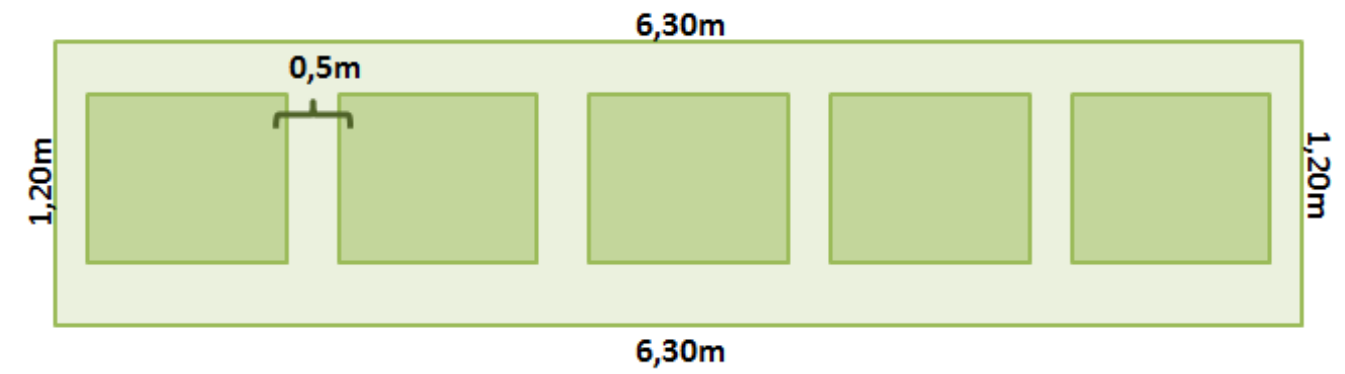

A Irrigação foi efetuada diariamente pelo período da manhã, durante os meses de estudo. A germinação foi considerada a partir da emissão radicular (figura 1) e acompanhada através de contagem diária por um período de 14 dias.

Para mensurar altura e diâmetro do coleto, utilizamos régua $30 \mathrm{~cm}$ e paquímetro digital com precisão de $0,1 \mathrm{~mm}$, marca MARBEG. A contagem de folhas foi efetuada manualmente. As medições foram feitas mensalmente sendo primeira medição foi no dia 29 de Março de 2016, e a segunda no dia 29 de Abril. 


\section{Tratamento e delineamento experimental}

O delineamento experimental utilizado neste trabalho inicial foi em blocos ao acaso com cinco tratamentos e cinco repetições de doze sementes cada, totalizando 300 sementes, houve um bloco acrescentado onde possuía 20\% a mais do total de 300 sementes, que serviriam para reposição de fins comerciais. Em cada bloco incluía os cinco tratamentos, distribuídos em cinco blocos (Figura 4).

Figura 4: Posicionamento dos tratamentos nos canteiros.

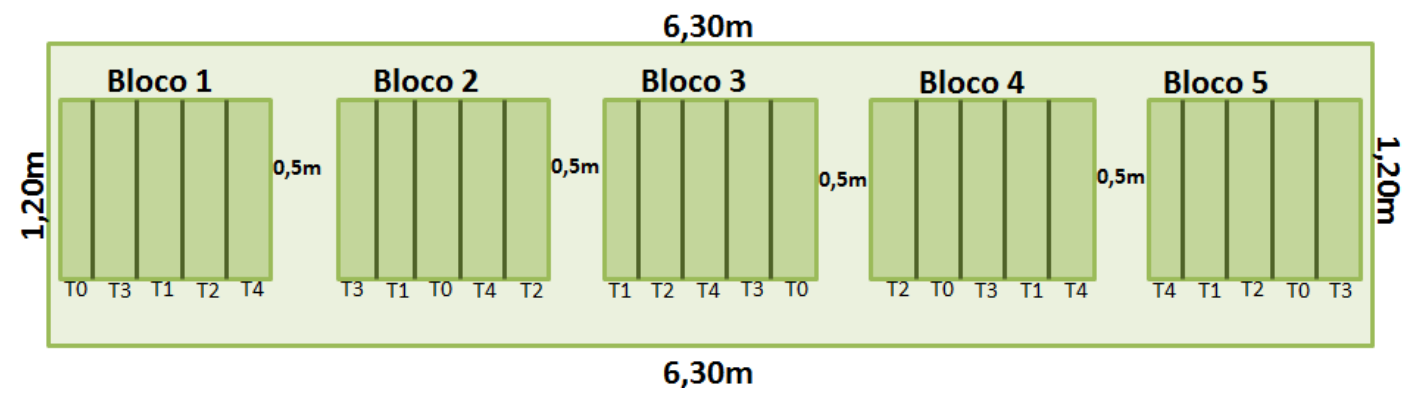

Sendo que os tratamentos são compostos da seguinte forma:

T0: Terra Preta (testemunha);

T1: Terra Preta + Serragem Curtida na proporção 2:1;

T2: Terra Preta + Serragem Curtida na proporção 3:1

T3: Terra Preta + Fibra de coco 2:1;

T4: Terra Preta + Fibra de coco 3:1;

\section{Biometria de frutos e sementes}

Para a biometria foram selecionadas ao acaso 100 unidades de sementes e frutos de pau preto. Foi feita a mensuração do comprimento, largura e espessura dos frutos e das sementes, com auxilio de um paquímetro digital com precisão de $0,1 \mathrm{~mm}$, da marca MARBEG. O comprimento foi medido da base até o ápice, e a largura e espessura medidas da porção mediana de ambos.

\section{Análise estatística}

Foi feito teste de normalidade, onde os dados deram normais de acordo com ShapiroWilk com índice acima de 0,05 usando programa de programa Action Stat. Análise de correlação foi feita no Excel. Foi calculado taxa germinativa (TG) com a fórmula:

TG: N/A x 100

Onde: $\mathrm{N}=$ numero de sementes germinadas 
$\mathrm{A}=$ numero total de sementes colocadas para germinar

O índice de velocidade de germinação (IVG) foi calculado com base nos dados de germinação diária, conforme a formula adaptada de POPINIGIS (1977):

$$
I V G=\frac{x 1}{y 1}+\frac{x 2}{y 2}+\frac{x 3}{y 3}+\cdots+\frac{x n}{y n}
$$

Onde: IVG: índice de velocidade de germinação;

$\mathrm{x} 1, \mathrm{x} 2, \ldots, \mathrm{xn}$ : número de sementes diárias germinadas;

$\mathrm{y} 1, \mathrm{y} 2, \ldots, \mathrm{yn}:$ dias transcorridos a partir da data da semeadura.

Foi realizada análise de variância (ANOVA) $(\mathrm{p}<0,05)$ analisando-se individualmente as variáveis: altura $(\mathrm{cm})$, diâmetro a altura do colo - DAC $(\mathrm{mm})$ e número de folhas. Aplicação de Tukey quando necessário. Foram realizadas análises estatística para se obter média, valor mínimo e máximo das variáveis, desvio padrão, erro padrão, variância da amostra, sendo os cálculos obtidos através do programa ASSISTAT.

Fórmula do delineamento em blocos:

$$
\mathrm{y}_{\mathrm{ij}}=\mu+\tau_{\mathrm{i}}+\beta_{\mathrm{j}}+\varepsilon_{\mathrm{ij}}
$$

onde $i=1,2, \ldots$, a tratamentos $j=1,2, \ldots, b$ blocos

$\mu$ é a média geral

$\tau$ i é o efeito fixo do i-ésimo tratamento

$\beta \mathrm{j}$ é o efeito fixo do j-ésimo bloco

вij é o erro aleatório.

\section{RESULTADOS E DISCUSSÃO}

A emergência das plântulas teve início a partir do quarto dia após a semeadura. As sementes de C. tocantinun Ducke possuem forma oblonga a circular, com marcas de cor marrom, circundando a extremidade; coloração marrom dourado, com pequenas manchas avermelhadas; hilo protuberante de cor marrom, em posição apical. Possui media de comprimento de $1,5 \mathrm{~cm}$, largura de $1,2 \mathrm{~cm}$, espessura $0,3 \mathrm{~cm}$. Isso esta de acordo com SILVA R.L.,2007 onde os resultados se assemelharam aos obtidos.

Os frutos são em vagem, deiscente, glabro, cor marrom escuro, superfície áspera, contendo em média 3 a 5 sementes por fruto, e médias de 9,16 cm de comprimento, 2,17cm de largura e 0,55cm de espessura. Esses dados estão de acordo com Lorenzzi (2003) (Tabela 1). 
Tabela 1. Mensuração de frutos e sementes de Cenostigma tocantinum Ducke (pau-preto). UFRA/ICA, Belém, 2016.

\begin{tabular}{lccc}
\hline Medidas Fruto & $\begin{array}{c}\text { Média cm } \\
(+-\mathbf{~ d p )}\end{array}$ & $\begin{array}{c}\text { Valor Mínimo } \\
\text { (cm) }\end{array}$ & Valor Máximo (cm) \\
\hline Comprimento $(\mathrm{cm})$ & $9,16(0,1)$ & 6,6 & 11,7 \\
Largura $(\mathrm{cm})$ & $2,17(0,16)$ & 1,9 & 2,6 \\
Espessura $(\mathrm{cm})$ & $0,55(0,06)$ & 0,3 & 0,7 \\
\hline & & & \\
\hline & & Valor & Valor Máximo (cm) \\
Medidas Semente & Média $(++-\mathbf{d p})$ & Mínimo $(\mathbf{c m})$ & 1,7 \\
\hline Comprimento $(\mathrm{cm})$ & $1,5(0,1)$ & 1,2 & 1,5 \\
Largura $(\mathrm{cm})$ & $1,2(0,1)$ & 0,9 & 0,5 \\
Espessura $(\mathrm{cm})$ & $0,3(0,04)$ & 0,3 & \\
\hline
\end{tabular}

Para análise de correlação do fruto, obteve resultados de correlação de comprimento e largura os índices foram classificados como moderada, pois o valor está entre 0,40 a 0,69, possuindo o valor de 0,58. A correlação de espessura e comprimento possui o grau de correlação de 0,25 classificada como fraca. Já largura e espessura são consideradas também como bem fraca no valor de 0,05 . O que significa que de acordo que o fruto cresce ele enlanguesce, e fica mais espesso.

As correlações de sementes mostram que os índices de largura e comprimento são moderados tendo índice de 0,45. Já espessura e comprimento apresentaram-se fraca com valor de 0.20. Sendo todos esses índices acima diretamente proporcionais, somente a correlação de largura e espessura da semente é inversamente proporcional, com valor de $-0,13$ em base a classificação Silvia E Shimakura 2006- (Tabela 2).

Para estes índices de correlação não necessita de análise de regressão, pois para estudos rigorosos o índice precisa ser igual ou maior de 0,6.

Tabela 2: Correlação de fruto e sementes em relação a medidas de comprimento, largura e espessura de Cenostigma tocantinum Ducke (pau-preto). UFRA/ICA, Belém, 2016.

\begin{tabular}{|ccc|}
\hline $\mathbf{R}$ & Semente & Fruto \\
\hline Espessura $\times$ Largura & $-0,13$ & 0,05 \\
\hline Espessura $\times$ Comprimento & 0,2 & 0,23 \\
\hline Comprimento $\times$ Largura & 0,45 & 0,58 \\
\hline
\end{tabular}

Já a taxa germinativa (TG) de todas as sementes foi de $68 \%$, de um total de 300 sementes. Já TG por tratamento foi de T0=41,67\%, T1=81,67\%, T2=76,66\%, T3 $=75 \%$ e T4=65\%, podendo afirma que nesse experimento o melhor substrato para germinação foi T1=Terra Preta + Serragem Curtida na proporção 2:1. 
Sendo índice de velocidade germinativa (IVG) para tratamento foi de $\mathrm{T} 0=$ 1,79, $\mathrm{T} 1=3,5, \mathrm{~T} 2=3,29, \mathrm{~T} 3=3,21, \mathrm{~T} 4=2,79$, (Tabela 3). SOUZA F.P. et al diz que o Índice de Velocidade de Germinação (IVG) é avaliado para obtermos uma resposta em relação ao vigor das mudas futuramente em campo. Nesse sentido obteve-se a melhor média de no índice de $T 1$, com IVG=3,5 com mudas provavelmente mais vigorosas e o pior índice foi no tratamento T0 com valor de 1,79 , com mudas que talvez sejam menos vigorosas.

Tabela 3: Taxa de germinação (TG \%) e do índice de velocidade de germinação (IVG) para tratamentos de Cenostigma tocantinum Ducke (pau-preto). UFRA/ICA, Belém, 2016.

\begin{tabular}{|ccc|}
\hline TRATAMENTOS & TG (\%) & IVG \\
\hline T0= TP & 41,67 & 1,79 \\
\hline T1 $=$ TP+ SC $2: 1$ & 81,67 & 3,5 \\
\hline T2 $=$ TP+ SC $3: 1$ & 76,66 & 3,29 \\
\hline T3= TP+FC 2:1 & 75,00 & 3,21 \\
\hline T4= TP+FC 3:1 & 65,00 & 2,79 \\
\hline
\end{tabular}

Onde: $\mathrm{TP}=$ Terra preta; $\mathrm{SC}=$ Serragem curtida; $\mathrm{FC}=$ Fibra de cocô.

As análises realizadas individualmente em relação à altura $(\mathrm{H})$, diâmetro do colo (DAC), não apresentam significância (Tabela 4; 5) , portanto podemos afirmar estatisticamente que suas médias são iguais (Tabela 7). Essa igualdade de média pode ser justificada pela aproximação das matrizes de coleta que estavam a menos de 100m uma da outra, com isso não obtendo uma variabilidade genética para H e DAC. Já o coeficiente de variação de $\mathrm{H}$ e DAC foi de $\mathrm{CV} \%=10.29, \mathrm{CV} \%=8.92$ respectivamente.

Tabela 4: Anova de altura (H) de Cenostigma tocantinum Ducke (pau-preto), onde não obteve-se significância. UFRA/ICA, Belém, 2016.

\begin{tabular}{|lcrcl|}
\hline FV & GL & \multicolumn{1}{c}{ SQ } & QM & F \\
\hline BLOCOS & 4 & 184.922 & 0.46231 & $0.2660 \mathrm{~ns}$ \\
\hline TRATAMENTO & 4 & 638.334 & 159.584 & $0.9181 \mathrm{~ns}$ \\
\hline RESIDUO & 16 & 2.780 .966 & 173.810 & \\
\hline TOTAL & 24 & 3.604 .222 & & \\
\hline
\end{tabular}

** significativo ao nível de $1 \%$ de probabilidade $(\mathrm{p}<.01)$

* significativo ao nível de $5 \%$ de probabilidade $(.01=<\mathrm{p}<.05)$

$\mathrm{ns}=$ não significativo $(\mathrm{p}>=.05)$

Tabela 5: Anova de diâmetro do colo (DAC) de Cenostigma tocantinum Ducke (pau-preto), onde se obteve não significância nos resultados. UFRA/ICA, Belém, 2016.

\begin{tabular}{|c|c|c|c|c|}
\hline FV & GL & SQ & QM & $\mathbf{F}$ \\
\hline BLOCOS & 4 & 0.37878 & 0.09470 & $1.5568 \mathrm{~ns}$ \\
\hline TRATAMENTO & 4 & 0.52318 & 0.13080 & $2.1502 \mathrm{~ns}$ \\
\hline RESIDUO & 16 & 0.97326 & 0.06083 & \\
\hline
\end{tabular}




\begin{tabular}{lll}
\hline TOTAL & 24 & 187.522
\end{tabular}

** significativo ao nível de $1 \%$ de probabilidade $(\mathrm{p}<.01)$

* significativo ao nível de $5 \%$ de probabilidade $(.01=<\mathrm{p}<.05)$

ns não significativo $(\mathrm{p}>=.05)$

Já análise de variância de número de folhas apresentou significância a 5 \% (Tabela6), onde pelo menos uma das medias se difere das demais. (Tabela 7). Com o CV\% 10.51. As medias que se diferenciam são a do T0 com valor de 4,31 por plântula. Apresentando assim o maior número de folhas, já as medias de T1, T2 e T3 são iguais entre si.

Tabela 6: Anova de quantidade de folhas de Cenostigma tocantinum Ducke (pau-preto), onde se obteve significância nos resultados. UFRA/ICA, Belém, 2016

\begin{tabular}{|lccll|}
\hline FV & GL & SQ & QM & F \\
\hline BLOCOS & 4 & 169.234 & 0.42308 & $2.6272 \mathrm{~ns}$ \\
\hline TRATAMENTO & 4 & 206.186 & 0.51546 & $3.2008^{*}$ \\
\hline RESIDUO & 16 & 257.666 & 0.16104 & \\
\hline TOTAL & 24 & 633.086 & & \\
\hline
\end{tabular}

** significativo ao nível de $1 \%$ de probabilidade $(\mathrm{p}<.01)$

* significativo ao nível de $5 \%$ de probabilidade $(.01=<\mathrm{p}<.05)$

ns não significativo $(\mathrm{p}>=.05)$

Tabela 7: Médias estatisticamente iguais em atura $(\mathrm{H})$ e diâmetro de colo (DC), e médias diferenciadas em número de folhas $\left(\mathrm{N}^{\circ} \mathrm{F}\right)$, de Cenostigma tocantinum Ducke (pau-preto), onde se obteve significância nos resultados. UFRA/ICA, Belém, 2016

\begin{tabular}{|llll|}
\hline Tratamento & Médias H & Médias DC & Médias $\mathbf{N}^{\circ} \mathrm{F}$ \\
\hline T0 $=$ TP & $13.69200 \mathrm{a}$ & $2.62200 \mathrm{a}$ & $4.31600 \mathrm{a}$ \\
\hline T1 $=$ TP+SC 2:1 & $12.86400 \mathrm{a}$ & $2.70800 \mathrm{a}$ & $3.49600 \mathrm{~b}$ \\
\hline T2 = TP+SC 3:1 & $12.80000 \mathrm{a}$ & $2.85400 \mathrm{a}$ & $3.68800 \mathrm{~b}$ \\
\hline T3= TP+ FC 2:1 & $12.14800 \mathrm{a}$ & $2.68200 \mathrm{a}$ & $3.64800 \mathrm{~b}$ \\
\hline T4= TP+ FC 3:1 & $12.57000 \mathrm{a}$ & $2.95800 \mathrm{a}$ & $3.94000 \mathrm{ab}$ \\
\hline
\end{tabular}

Onde: $\mathrm{TP}=$ Terra preta; $\mathrm{SC}=$ Serragem curtida; $\mathrm{FC}=$ Fibra de cocô.

\section{CONCLUSÃO}

Constatou-se que as mudas de Cenostigma tocantinum Ducke apresentaram resposta em todos os tratamentos com relação ao desenvolvimento inicial das plântulas, porem o T1, serragem curtida 2:1 foi o que mais se destacou, com melhor qualidade e maior porcentagem de germinação das mudas. Sendo os mais indicados para a produção de mudas com qualidades.

Os indivíduos na qual se obteve baixos índices de germinação foi o T0, a testemunha. Os fatores podem ter sido por estar no fim do período de dispersão ou a 
influência do material genético das sementes por ser desconhecido, com isso afetando de forma negativa o desenvolvimento das mudas.

Se tiver um bom conhecimento da espécie e planejamento dos substratos a utilizar é primordial para o sucesso do plantio, pois garante poucas perdas no processo de germinação e uma boa interação que relacione espécies e substratos, visando o melhor desenvolvimento.

\section{REFERÊNCIAS}

BARROSO, G. M.; Sistemática de Angiosperma do Brasil. Minas Gerais: Impressa Universitária, v. 2, p. 337, 1991.

BRAGA JUNIOR, J. M.; BRUNO, R. L. A.; ALVES, E. U. Emergência de plântulas de Zizyphus joazeiro Mart. (Rhamnaceae) em função de substratos. Revista Árvore, v.34, n.4, p.609-616, 2010.

DESAI, B. B.; KOTECHA, P. M.; SALUNKHE, D. K. Seeds handbook Biology, Production, Processing and Storage. 1 ed. New York: Basel, 1997, 627p.

GARCIA, L.C.; MORAES, R.P. de; LIMA, R.M.B. de Determinação do Grau Crítico de Umidade em Sementes de Cenostigma tocantinum Ducke. Revista Brasileira de Sementes, v. 30, n 3, p. 172-176, 2008

LIMA, H. C.; CORREIA, C. M. B.; FARIAS, D. S. Leguminosae. In. M. P. M. DE LIMA, R. R, GUEDES-BRUNI. Revista Ecológica de Macaé de Cima: Nova Friburgo-Rj; Aspectos Florísticos das Espécies Vasculares. Jardim Botânico Rio de Janeiro, Rio de Janeiro, p. 167228, 1994.

LOPES, J. C.; PEREIRA, M. D. Germinação de sementes de cubiu em diferentes substratos e temperaturas. Revista Brasileira de Sementes, v.27, n.2, p.146-150, 2005.

LORENZI, H. Árvores brasileiras: manual de identificação e cultivo de plantas arbóreas nativas do Brasil. / Harri Lorenzi. 2 ed. Nova Odessa, SP: Edit. Pantarum. V. 2, 368 p. 199

Gusmão M. T. A.; Ferreira G. C.; Ohashi S. T. Espécies Florestais Nativas e Exóticas na Amazônia Oriental. Belém - PA. p. 57, ISBN 978-85-7295-075-6

PEIXOTO, J. R. Efeito da matéria orgânica, do superfosfato simples e do cloreto de potássio na formação de mudas de maracujazeiro-azedo (Passiflora edulisf. flavicarpa ). 
1986. 101f. Dissertação (mestrado em Agronomia) - Universidade Federal de Lavras, Lavras, 1986.

SANTOS, P. C. T. C.; VIEIRA, L. S.; VIEIRA, M. N. F.; CARDOSO, A. Os solos da Faculdade de Ciências Agrárias do Pará. Belém: FCAP, 1983. 60 p. (FCAP, Informe Didático, 5).

SILVA, R. P. da.; PEIXOTO, J. R.; JUNQUEIRA, N. T. V. Influência de diversos substratos no desenvolvimento de mudas de maracujazeiro-azedo (Passiflora edulis Sims f. flavicarpa DEG). Revista Brasileira de Fruticultura, Jaboticabal-SP , v.23, n.2, p.377-381, agosto 2001

SILVA, R. L. Fenologias em ambiente urbano, morfologia de semente e da plântula e germinação sob condições adversas de Cenostigma tocantinum Ducke. Dissertação apresentada ao programa de pós graduação em Biologia Tropical, Manaus, Amazonas, 2007.

SOUZA, E. R. B.; CARNEIRO, I. F.; NAVES, R. V.; BORGES, J. D.; LEANDRO, W. M.; CHAVES, L. J. Emergência e crescimento de cagaita (Eugenia dysenterica DC.) em função do tipo e do volume de substratos. Pesquisa Agropecuária Tropical, v.31, n.2, p.iMN89-95, 2001.

$\begin{array}{llll}\text { Sousa } & \text { F.P.; } & \text { Oliveira } \quad \text { G.M.R;Martins } \quad \text { W.B.R.; Vale } \quad \text { R.S.V.,CorreiaR.G. }\end{array}$ DESENVOLVIMENTO INICIAL DE MUDAS DE PARICÁ (Schizolobium Amazonicum Huber ex. Ducke) DE DIFERENTES PROCEDÊNCIAS SUBMETIDAS A PERÍODOS DE IRRIGAÇÃO.

Silvia; Shimakura disponível em:< http://leg.ufpr.br/ silvia/CE003/node74.htm>l. Interpretação do coeficiente de correlação2006-08-30 Acessado em: 18 de maio de 2016[ 